\title{
Stochastic Resonance Phenomenon of Bistable System Driven by Binary Phase Shift Keying Signal under the Alpha Stable Noise
}

\author{
Gaohui Liu, Te Li \\ School of Automation and Information Engineering, Xi'an University of Technology, Shaanxi,China \\ liugh68@xaut.edu.cn
}

Keywords: Stochastic resonance, binary phase shift keying signal, alpha stable noise, SNR gain Abstract. Many problems in communication and Radar signal processing involve the detection of low signal to noise ratio (SNR) signal in non-Gaussian noise environment. The stochastic resonance of the nonlinear bistable systems driven by binary phase shift keying signal under the alpha stable distributed noise was investigated. Firstly, the detection model of nonlinear bistable stochastic resonance system was constructed, driven by binary phase shift keying (BPSK) signal and alpha stable noise. Then, the effect of the Alpha stable noise intensity and system parameters on the output signal-to-noise ratio(SNR) and SNR gain were explored. The results show that separate adjustment of system parameters $a$ and $b$ or noise parameters $m$ can produce stochastic resonance phenomenon and achieve enhancement of the input signal. Moreover, existence the optimal values of $a, b$ and $m$ can be make the output of the system resonance is the strongest, which can effectively detect the weak input signal.

\section{Introduction}

With the rapid development of wireless communication technology, various kinds of communication signal modulations and signal systems continuously are constantly being applied, it will directly lead to the signal intensity of increasing in the limited space of the communication spectrum and cause spectrum congestion and overlap, coupled with the complex and changeable external noise interference, this makes the wireless communication receiver signal detection is facing severe challenges ${ }^{[1]}$.At present, the signal detection methods are mostly to suppress the noise for the purpose $^{[2-4]}$,When the noise is near or coincident with the frequency of the signal, this method is difficult to meet the actual requirements. The stochastic resonance(SR) method proposed by Benzi is not to directly eliminate noise, but to use the noise to enhance the ordered output of the signal ${ }^{[5,6]}$.With the cooperative effect of nonlinear system, input signal and noise, the signal to noise ratio of the output of system will be increased, due to the conversion of the noise energy to the useful signal.This advantage makes the stochastic resonance has been widely used in the fields of biological, chemical, electronics, image processing and so on.

The current research of stochastic resonance is mainly aimed at the Gauss noise ${ }^{[7-9]}$, but in practical applications a lot of noise has significant pulse characteristics and smear characteristic, at this moment, it can not be used to describe Gaussian distribution. Therefore, this paper introduces alpha stable noise to describe these signals, alpha stable distribution can keep the natural noise generation mechanism and propagation conditions, and can be consistent with actual observation data, and has more extensive applicability ${ }^{[10,13,15]}$. In recent years, some scholars have studied the SR phenomenon under the background of stable distribution noise ${ }^{[11-15]}$.Lingzao Zeng studied aperiodic SR phenomenon under the Lévy noise ${ }^{[11]}$. Wenying Zhang et al uses SR theory to detect the signal in Lévy noise $^{[12]}$.Guangli Zhang et al studied parameter-induced SR phenomenon under the alpha stable noise ${ }^{[13]}$.Shangbin Jiao et al studied the SR detection method of high frequency and low frequency weak signals under stable noise ${ }^{[14,15]}$. But these studies are based on the periodic signal, However, there is no report on the study of the SR of the BPSK signal under the alpha stable noise. Therefore, this paper on the basis of previous studies, mainly explores nonlinear bistable SR of BPSK signal in the alpha stable noise environment. 


\section{Bistable Stochastic Resonance System Model under the Background of Alpha Stable Noise}

Alpha Stable Distribution. Alpha stable distribution is also known as the Lévy partial alpha stable distribution. It is the only meet the distribution of Generalized Central Limit Theorem, and is the generalization of Gaussian distribution ${ }^{[13]}$. It is described by using the characteristic function ${ }^{[10,11,16]}$, which can be expressed as

$$
\varphi(t)=\exp \left\{j \mu t-\gamma|t|^{\alpha}[1+j \beta \operatorname{sgn}(t) \omega(t, \alpha)]\right\}
$$

Where $\operatorname{sgn}(t)=\left\{\begin{array}{ll}1, & t>0 \\ 0, & t=0 \\ -1, t<0\end{array}\right.$ is the sign function and $\omega(t, \alpha)=\left\{\begin{array}{ll}\tan \frac{\alpha \pi}{2}, & \alpha \neq 1 \\ \frac{2}{\pi} \lg |t| & \alpha=1\end{array}\right.$. From this, the stable distribution is completely determined by four parameters $\alpha, \beta, \gamma$ and $\mu$. Where $\alpha \in(0,2]$ is characteristic exponent, which is used to measure the thickness of the tail distribution function; $\beta \in[-1,1]$ is the symmetry parameter, when $\beta=0$, the stable distribution is symmetrical about the position parameters; $\gamma \in[0,+\infty)$ is the proportional parameter, which is similar to the variance of the Gauss distribution, in the case of Gaussian is equal to half of the variance; and $\mu \in(-\infty,+\infty)$ is the positional parameters.

Bistable stochastic resonance system model and numerical solution. The system Langevin Equation under the combined action of alpha stable noise and input signals can be expressed as ${ }^{[5,14]}$ :

$$
d x / d t=-U^{\prime}(x)+s(t)+\Gamma_{\alpha}(t)
$$

Where $U(x)=-(a / 2) x^{2}+(b / 4) x^{4}$ is the potential function of the bistable system, $a$ and $b$ are system parameters, $s(t)$ is the input signal; $\Gamma_{\alpha}(t)=m \eta(t)$ represents alpha stable noise, $m$ is the noise intensity.

When the input signal and the alpha stable noise are zero, the bistable system has two stable equilibrium states in $x= \pm \sqrt{a / b}$ and $\Delta U=a^{2} / 4 b$ is the barrier height. When the input signal and noise are added to the nonlinear bistable system, a part of the noise energy is converted into the signal energy under the matching condition, so as to improve the output SNR, which can effectively extract the weak signal in the noise background.

This paper is mainly based on the fourth-order Runge-Kutta method ${ }^{[13,18]}$ to solve the formula (2), and combined with the characteristics of the input signal and noise to obtain the standard four-order Runge-Kutta solution,as follows:

$$
\left\{\begin{array}{l}
k_{1}=h\left(a x(i)-b x^{3}(i)+s(i)\right) \\
k_{2}=h\left(a\left(x(i)+\frac{k_{1}}{2}\right)-b\left(x(i)+\frac{k_{1}}{2}\right)^{3}+s(i)\right) \\
k_{3}=h\left(a\left(x(i)+\frac{k_{2}}{2}\right)-b\left(x(i)+\frac{k_{2}}{2}\right)^{3}+s(i)\right) \\
k_{4}=h\left(a\left(x(i)+k_{3}\right)-b\left(x(i)+k_{3}\right)^{3}+s(i)\right) \\
x(i+1)=x(i)+\frac{1}{6}\left(k_{1}+2 k_{2}+2 k_{3}+k_{4}\right)+\Gamma_{\alpha}(i) h 1 / \alpha
\end{array}\right.
$$

Where $h$ is sampling step, $a$ and $b$ are system parameters, $x(i)$ is the $i$ times sampling value of input signal; $\Gamma_{\alpha}(i)$ is the $i$ times sampling value of alpha stable noise.

Stochastic resonance measurement method. Since the alpha stable distribution does not have a finite second moment, the long jump of particle with the decrease of the alpha stability index can make the path change regional infinity. In order to make a theoretical analysis in this noise environment, it is necessary to try to suppress the influence of noise pulse.Therefore, this paper uses the symbolic function to limit the magnitude or artificially truncated of observations data ${ }^{[13,14]}$,therefore, the problem of particle jumping trajectory infinite is solved, i.e., $x(t)=10 \operatorname{sgn}(x(t))(x(t)>10)$.In this way, we can calculate the finite second moment of alpha stable distribution. 
In this paper, SNR and SNR gain are used as the main indicators to measure the output enhancement of nonlinear bistable system ${ }^{[13,15,19]}$, where the SNR gain is defined as the ratio of the output SNR and the input $\mathrm{SNR}^{[14,19]}$.

\section{Simulation and Analysis}

Influence of System Structure Parameters on Bistable Stochastic Resonance Output.The alpha stable noise parameters are set to $\alpha=1.2, \beta=-1,0$ and $1 ; \gamma=1, \mu=0 ; m=0.63$. With the BPSK signal as input signal, the symbol rate is set to $10 \mathrm{~B}$, the carrier frequency is set to $50 \mathrm{~Hz}$. The system parameter is set to $b=0.1$ to carry out simulation experiment,The SNR and G with the change of the parameter $a$ of the curve are obtained, the simulation results are shown in Fig. 1 and Fig. 2.

Fig. 1 is the output SNR of system along with the change of the system parameter $a$. It can be seen that the output SNR curve with the increase of system parameter $a$ is non-monotonic change process. This explains that the system output has a number of enhanced interval or enhancement points, that is to say, the nonlinear bistable SR system has non-single resonance interval or resonance point, Just these resonance interval or resonance point to improve the range of the system output is different. For example, the figure of the resonance interval $(0,20)$ and $(95,150)$ compared to $(20,50)$ and $(70,95)$,the SNR of the former is relatively large,And there are a plurality of resonance points in these intervals, but there is an optimal resonance point. And the position of the resonance interval or the resonance point is not changed with the change of the symmetric parameter $\beta$,basically remain in the same position on the interval,and the occurrence of resonance point is relatively concentrated. The synergistic effect of the input signal and noise, and stable nonlinear bistable system can achieve a good match in this interval, so that the output of the useful signal can be significantly enhanced.

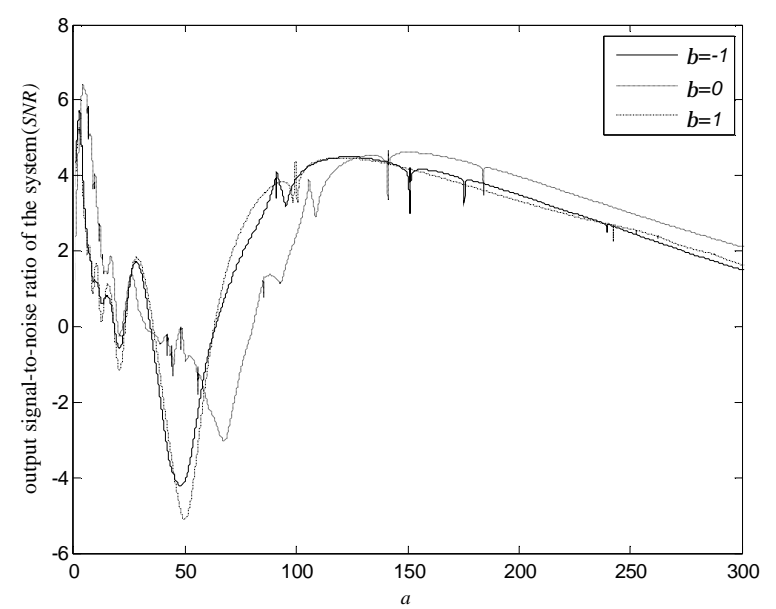

Fig. 1.The Curve of Output SNR with System Parameter $a$

Fig. 2 is the output SNR gain graph of system under different symmetry parameters along with the change of the system parameter $a$. In this paper, the SNR gain is to seek the average of the twenty experiments and drawn. From this graph, it can clearly reflect the output SNR also presents non-monotonic change process. This shows that the system has the resonance interval and the optimal resonance point when the whole system reaches stochastic resonance. Compared with the input that can reflect the current parameters of the system output to improve the situation. When the system parameter $b$ is 0.1 , the optimal values of $a$ in the resonance interval are $2.4,3,6,10.2,15.8,30.1,124$, 181.5. In terms of the resonance effect of the whole bistable system, the SR effect is the most prominent when the $a$ is 2.4 . The output increase is 2.75 times of the original,but this is only when the noise parameter is set to $\alpha=1.2, \beta=-1,0,1 ; \gamma=1, \mu=0 ; m=0.63$, and the system parameter $b$ is set to 0.1 , the optimal value of SNR is improved by the bistable stochastic resonance system.Of course, the SNR gain will gradually increase through adjusting the system parameters and noise parameters.Here it is just reflects the effect of system parameter $a$ on the output of the whole stochastic resonance system. 


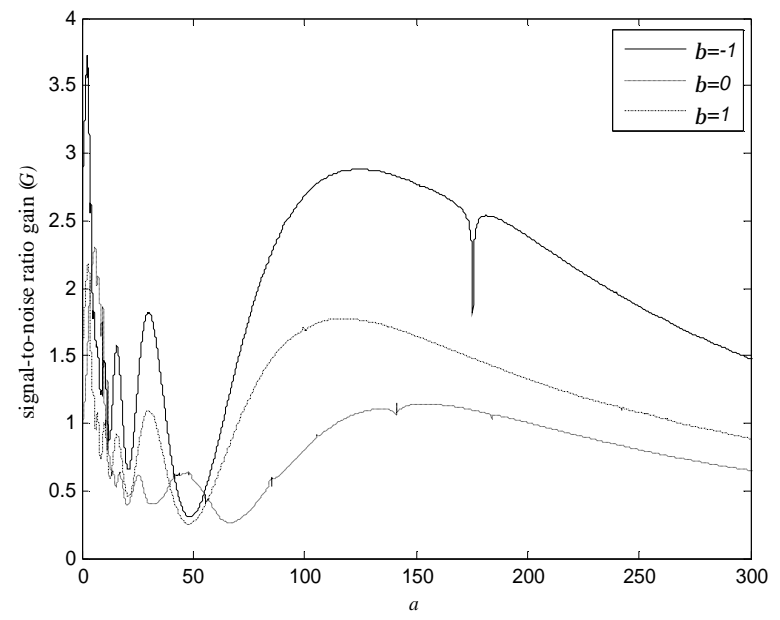

Fig. 2. The Curve of SNR Gain with System Parameter $a$

In order to distinguish the effect between system parameter $b$ and the output of the bistable stochastic resonance system, the system parameter $\mathrm{A}$ is 57.57 , and the other parameters remain unchanged, We obtain the output SNR graph along with the change of system parameter $b$, as shown in Figure 3. It can be seen from the figure 3, the system output SNR with the change of $b$ remain stable at $b<0.5$.But the output SNR with increasing $b$ also exhibit non-monotonic variation,this is similar to the rule of system parameter $a$ to the system output.The bistable SR system also has non-single non-single resonance interval and resonance point. When $b<0.8, \beta=0$, the resonance interval is $(0.5,0.62)$ and $(0.64,0.72)$ as well as $(0.72,0.79), \beta=1$ the resonance interval is $(0.66,0.79)$, $\beta=-1$ the resonance interval is $(0.77,0.79)$. When $b>0.8$, the resonance interval is $(0.8,0.86)$, $(0.95,0.98)$ and $(0.98,1.04)$, and the position of the resonance interval or the resonance point is not changed with the change of the symmetric parameter $\beta$,basically remain in the same position on the interval,and the occurrence of resonance point is relatively concentrated. The synergistic effect of the input signal and noise, and stable nonlinear bistable system can achieve a good match in this interval, so that the output of useful signal can be significantly enhanced.

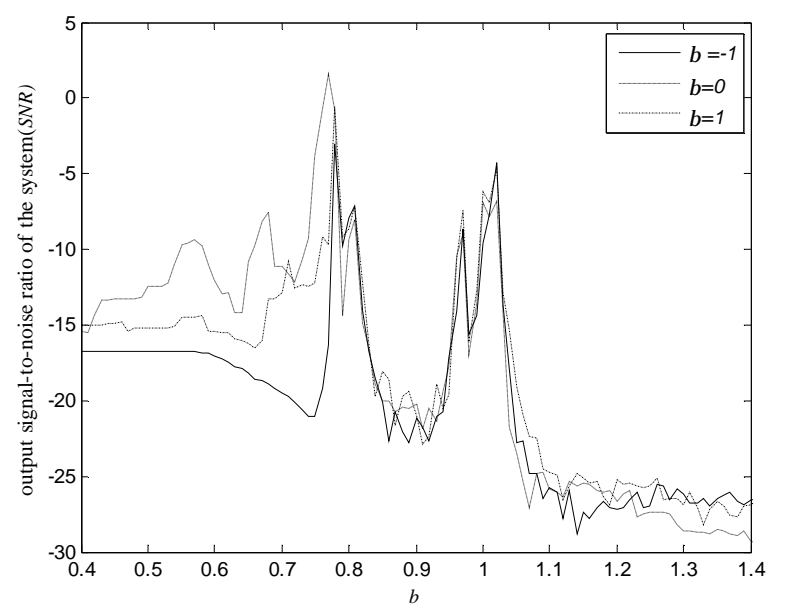

Fig. 3. The Curve of Output SNR with system parameter $b$

Fig. 4 is the system SNR gain graph under different symmetry parameters along with the change of the system parameter $b$, the graph can clearly reflect that the optimal value of $m$ within the interval of each resonance can be obtained when $\beta$ is equal to -1 and the system parameters $b$ is equal to 0.78 , $0.81,0.97,1.02$; the optimal value of $m$ within the interval of each resonance can be obtained when $\beta$ is equal to 0 and the system parameters $b$ is equal to $0.57,0.67,0.77,0.81,0.97,1.01$; the optimal value of $m$ within the interval of each resonance can be obtained when $\beta$ is equal to 0 and the system parameters $b$ is equal to $0.68,0.78,0.81,0.97,1.02$; And in terms of the resonance effect of the 
whole bistable system, the SR effect is best when the $b$ is about 1.02. It also reflects that the position of the system output enhancement does not change with the change of the symmetry parameter $\beta$ when the $b$ reaches a certain range(i.e., $b>0.8$ ).

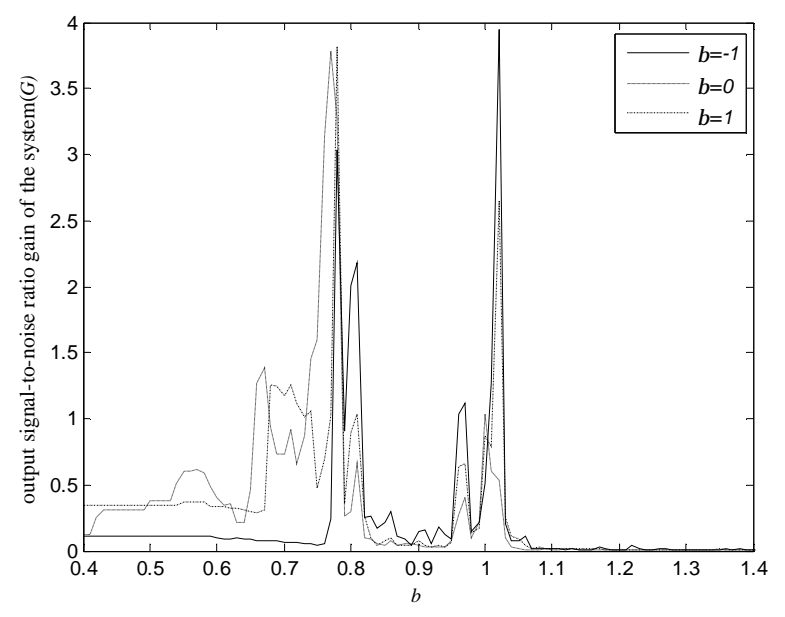

Fig. 4. The Curve of SNR Gain with system parameter $b$

In summary, by adjusting system parameters to achieve the stochastic resonance, the value of $a$ and $b$ is not the only, there are multiple resonance interval, $a$ and $b$ have optimal values in the resonance range. If the system parameters are selected properly, the SR phenomenon can be achieved, which can be detected the useful signal in the stable noise.

Influence of Alpha Noise Intensity $m$ on Bistable Stochastic Resonance Output. With the BPSK signal as input signal, the symbol rate equals $10 \mathrm{~B}$, the carrier frequency is set $50 \mathrm{~Hz}$, the alpha stable noise parameters are set to $\alpha=1.2, \beta=-1,0$ and $1 ; \gamma=1, \mu=0 ; m=0.63$. Then maintain the system parameter $a=52.48, b=0.01$ to carry on the simulation experiment. The SNR and SNR Gain simulation graph with the change of the alpha noise intensity $m$ are obtained, As shown in Fig. 5 and Fig. 6. Fig. 5 is the system output SNR graph along with the change of the alpha noise intensity $m$. It can be seen that the output SNR presents non-monotonic variation, which indicates the existence of the optimal value of $m$ for stochastic resonance.

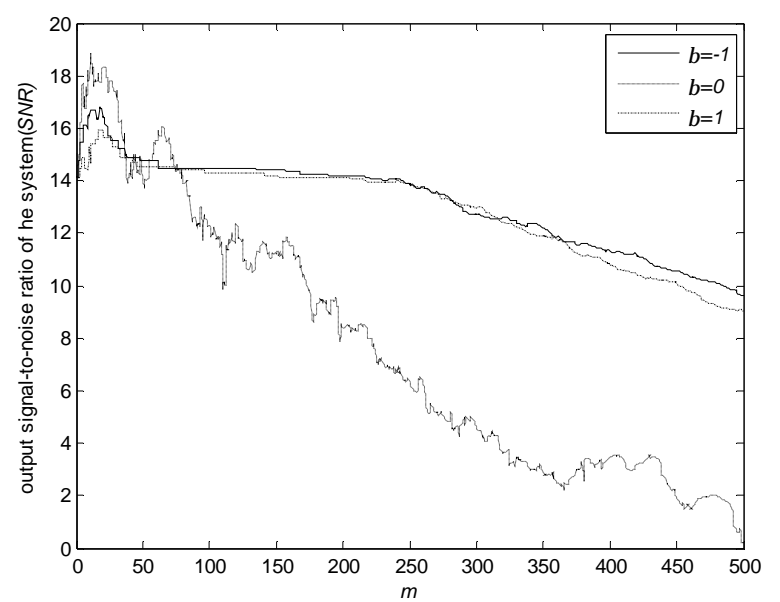

Fig. 5. The Curve of Output SNR with Noise Intensity $m$

With the increase of the noise parameter $m$, the output SNR of system is firstly increased and then decreased ,It is showed that there exists a single resonance interval $(0,45)$ in the nonlinear bistable stochastic resonance system, and the existence of the optimal value of $\mathrm{m}$ can significantly enhance the output of the system. And this resonance range is not changed with the change of the symmetry parameters $\mathrm{m}$,also it reflects the alpha stable noise plays a role in promoting the output of the whole nonlinear bistable system in this interval, it can also achieve SR phenomenon by separately adjusting the alpha stable noise intensity $m$, so as to achieve the purpose of improving the output of the system. 


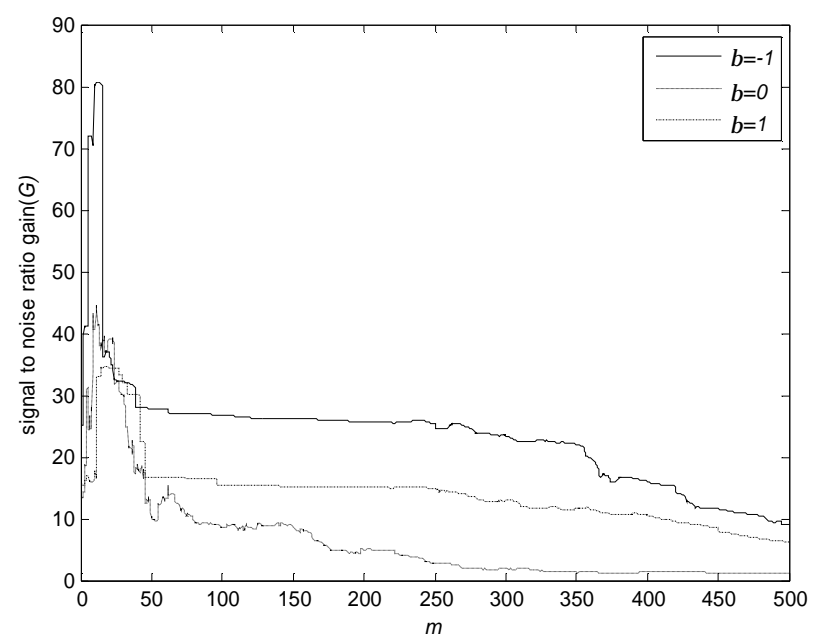

Fig. 6. The Curve of SNR Gain with Noise Intensity $m$

Fig. 6 is the SNR gain graph under different symmetry parameters along with the change of the alpha stable noise intensity $m$.With increasing noise parameters, the output signal noise ratio gain of the system also presents non-monotonic change process, And the output signal noise gain of the system is firstly increased and then decreased, and there exists a maximum peak value. When the symmetry parameter $\beta$ equals -1 , noise intensity $m$ is equal to 5.4 , there is a peak value; when $\beta$ is equal to $0, m$ is equal to 5.5 , there is a peak value and also there exists a peak when $\beta$ is equal to $1, m$ equals 5.6. This shows that for the corresponding system parameters and noise parameters, these three points can make the resonance effect of the whole bistable system is the most prominent, and it also shows that the optimal value of the noise intensity when the signal to noise ratio gain of the system output is the strongest is not changed with the change of the symmetric parameter $\beta$.Of course, the output of the whole stochastic resonance system will be further improved by further reasonable adjustment of the system parameters .

\section{Conclusions}

In this paper, the nonlinear bistable stochastic resonance phenomenon of binary phase shift keying signal in the alpha stable distributed noise environment has been studied, and the detection model of nonlinear stochastic resonance system driven by alpha stable noise and BPSK signal is constructed, and the effect of the Alpha stable noise intensity and system parameters on the output signal-to-noise ratio(SNR) and SNR gain have been explored. The following conclusions can be obtained through the above research: 1) By adjusting the system parameters, the use of the coordinating role of the input of BPSK signal, alpha noise stable and the bistable system can achieve the stochastic resonance, and the input BPSK signal has a significantly enhanced role; 2) Adjusting system parameters to realize stochastic resonance, the output of system has non-single stochastic resonance interval or resonance point, Just these resonance interval or resonance point to improve the range of the system output is different.The system parameters $a$ and $b$ have the best alue, when it reaches a certain value, it does not change with the change of symmetric arameter $\beta ; 3$ ) With the increase of the alpha noise parameter $m$, the output SNR and SNR gain of system is firstly increased and then decreased, it is showed that there exists the optimal value of $m$ can make the resonance effect of the output in the case of different symmetry parameters. These conclusions have a certain reference value for parameter-induced stochastic resonance and alpha stable noise induced stochastic resonance.

\section{Acknowledgements}

This work was financially supported by the National Natural Science Foundation of China (61671375). 


\section{References}

[1] Liping Wu.The key technologies about signal dectection and estimation under complex electromagnetic environment[D]. Xi'an: Xidian University,2011(in Chinese).

[2] Jinzhan Gao. Weak signal detection (Second Edition) [M]. Beijing: Tsinghua University press, 2011(in Chinese).

[3] M. I. plett.Transient deteetion with cross wavelet transforms and wavelet Coherence[J]. IEEE Transactions on Signal Proeessing, 2007, 55(5):1605-1611.

[4] Deyun Wei, Qiwen Ran,Yuanmin Li. Generalized sampling expansion for bandlimited signals assoeiated with the fractional Fourier transform[J]. IEEE Signal Proeessing Letters, 2010, 17 (6) :595-598.

[5] Benzi R,Sutera A,Vulpiania A. The Mechanism of Stochastic Resonance [J]. J Phys A: Math Gen,1981,14(11):L453-L457.

[6] Benzi R,Parisi G,Sutera A,et al. A Theory of Stochastic Resonance in Climate Change [J]. SIAM Journal on Applied Mathematics, 1983, 43(3):565-578.

[7] Jijun Tong, Guanglei Zhang, Qiang Cai , et al. Application of threshold stochastic resonance in low concentration gas detecting[J]. Journal of Zhejiang University (Engineering Science), 2015,49 (1): 15-19(in Chinese).

[8] Tingfeng Ming, Yongxiang Zhang,Jing Li.Parameter self-optimizing method and its application of stochastic resonance model[J].Journal of Tianjin University(Science and Technology), 2014, 47(10):886-891(in Chinese).

[9] Weijiao Fan, Fuzhong Wang, Guanglu Zhang. Detecting weak signals by combining stochastic resonance with filter[J].Journal of Applied Acoustics,2015,34(2):169-174(in Chinese).

[10] Weichao Yang, Chun-hui Zhao, Bao-zhi Cheng. Recognition of communication signals in noise with alpha stable distribution[J].Journal of Applied Sciences,2010,28(2):111-114(in Chinese).

[11] Lingzao Zeng. The theory of aperiodic stochastic resonance in anomalous process[D].Hangzhou: Zhejiang University, 2008(in Chinese).

[12] Wenying Zhang, Zili Wang,Weidong Zhang. Signal detection from Lévy noise via stochastic resonance[J].Control Engineering of China,2009,16(5):638-640(in Chinese).

[13] GuangLi Zhang ,XiLu Lü , YanMei Kang.Parameter-induced stochastic resonance in overdamed system with $\alpha$ stable noise[J].Acta Phys. Sin.,2012,61(4):040501(in Chinese).

[14] ShangbinJiao, Chao Ren, Weichao Huang, Yanming Liang. Parameter-induced stochastic resonance in multi-frequency weak signal detection with $\alpha$ stable noise[J]. Acta Phys. Sin., 2013, 62(21): 210501(in Chinese).

[15] ShangBin Jiao, Rong Yang,Qing Zhang,et al.Stochastic resonance of asymmetric bistable system with $\alpha$ stable noise[J].Acta Phys. Sin.,2015,64(2):020502(in Chinese).

[16] A Janicki, A Weron. Simulation and Chaotic Behavior of $\alpha$ Stable Stochastic processes[M]. Marcel Dekker, New York, 1994.

[17] B. McNamara, K. Wiesenfeld. Theory of stochastic resonance[J]. Physical Review A, 1989,39 (9) : 4854-4869.

[18] Xingguo Jiang, Jinhai Xu, Long Zhang . Real-time processing method based on stochastic resonance for inertial sensor signals[J].Systems Engineering and Eletronics, 2014,36(11):2280-2287 (in Chinese).

[19] Niaoqing Hu.Theory and method of stochastic resonance weak characteristic signal detection [M]. Beijing: National Defence Industry Press, 2012(in Chinese). 